\title{
Performance Bounds for Cooperative Simultaneous Localization and Mapping (C-SLAM)
}

\author{
Anastasios I. Mourikis and Stergios I. Roumeliotis \\ Dept. of Computer Science \& Engineering, University of Minnesota, Minneapolis, MN 55455 \\ Email: \{mourikis|stergios\}@cs.umn.edu
}

\begin{abstract}
In this paper we study the time evolution of the position estimates' covariance in Cooperative Simultaneous Localization and Mapping (C-SLAM), and obtain analytical upper bounds for the positioning uncertainty. The derived bounds provide descriptions of the asymptotic positioning performance of a team of robots in a mapping task, as a function of the characteristics of the proprioceptive and exteroceptive sensors of the robots, and of the graph of relative position measurements recorded by the robots. A study of the properties of the Riccati recursion which describes the propagation of uncertainty through time, yields (i) the guaranteed accuracy for a robot team in a given C-SLAM application, as well as (ii) the maximum expected steady-state uncertainty of the robots and landmarks, when the spatial distribution of features in the environment can be modeled by a known distribution. The theoretical results are validated by simulation experiments.
\end{abstract}

\section{INTRODUCTION}

In order for a multirobot team to coordinate while navigating autonomously within an area, all robots must be able to determine their positions with respect to a common frame of reference. In an ideal scenario, each robot would have direct access to measurements of its absolute position, such as those provided by a GPS receiver, or those inferred by detecting previously mapped features. However, reliance on GPS is not feasible in a number of situations, since GPS signals are not available everywhere (e.g., indoors), or, triangulation techniques based on them may provide erroneous results due to multiple reflections (e.g., in the vicinity of tall structures and buildings). Moreover, compiling a detailed map of the environment is a tedious and time-consuming process, while numerous applications require robots to operate in unknown surroundings, whose structure cannot be determined in advance. This suggests that additional means are required for aiding odometry when groups of mobile robots localize.

In situations where absolute position information is not available, the robots of a team can still improve their localization accuracy, by recording robot-to-robot relative position measurements, and processing them in order to update their position estimates (e.g., [1], [2], [3]). This method results in a substantial improvement in estimation accuracy, compared to simple Dead-Reckoning localization schemes. However performing Cooperative Localization (CL) solely based on relative position measurements has the limitation that the uncertainty of the robots' position estimates continuously increases, and the attained accuracy may not be sufficient for certain applications. An alternative approach is for the robots to localize while concurrently building a map of the environment, in which case the uncertainty in their position estimates remains bounded [4]. This introduces the problem of Cooperative Simultaneous Localization And Mapping (C-SLAM) that has recently attracted the interest of many researchers. The number of potential applications that require robots to perform C-SLAM is continuously growing, as autonomous vehicles are employed for tasks ranging from planetary exploration and environmental monitoring, to construction and transportation.

In this work, C-SLAM is considered within the Stochastic Mapping framework [5], [6]. We assume that the mobile robots move continuously and randomly in a planar environment, while recording measurements of the relative positions (i.e., range and bearing) of other robots in the team, and of point landmarks that exist in the environment. A means of describing the exteroceptive measurements that are recorded at each time step is the associated Relative Position Measurement Graph (RPMG), i.e., the graph whose vertices represent the robots and landmarks, while its directed edges correspond to the robot-to-robot and robot-to-landmark measurements (cf. Fig. 1(a)). We impose the constraint that the RPMG is a connected graph, i.e., that there exists a path between any two of its nodes. This constraint arises naturally and is not a restrictive one, since if an RPMG is not connected, then it can always be decomposed into smaller, connected sub-graphs. Each of these sub-graphs corresponds to an isolated group of robots and/or landmarks, whose position estimation problem can be studied independently.

This paper presents the first derivation of analytical upper bounds on the positioning uncertainty during C-SLAM for a possibly heterogeneous team of mobile robots navigating within a 2D environment populated with point features. The metric used to describe the localization uncertainty is the covariance matrix associated with the errors in the position estimates for the robots and the mapped features. The closedform expression of Lemma 4.3 establishes the guaranteed accuracy attainable by a robot team in a given mapping application, as a functional relation of the noise parameters of the robots' sensors and the topology of the RPMG. Furthermore, the result of Lemma 4.5 demonstrates how prior information about the spatial density of landmarks can be utilized in order to compute a tight upper bound on the expected covariance of the positioning errors. To the best of our knowledge, the proposed bounds constitute the only existing analytical tools for predicting the mapping precision, 
as well as the accuracy of the robots' localization in a given CSLAM application. Hence, they facilitate the selection of the required sensor parameters, in order to satisfy task-imposed performance constraints.

\section{RELATED WORK}

Most of the existing approaches to both single- and multirobot SLAM have been inspired by the seminal papers of Moutarlier and Chatila [6] and of Smith, Self, and Cheeseman [5], [7] that introduced the notion of the Stochastic Map and emphasized the importance of properly accounting for the correlations between all the robots' and landmarks' position estimates. However, maintaining all the cross-correlation elements of the covariance matrix in EKF-based SLAM results in algorithms with computational complexity quadratic in the size of the state vector. Thus, the majority of subsequent research on SLAM has focused on devising scalable algorithms, that achieve performance comparable to that of an EKF-based approach to SLAM that accounts for the cross-correlation terms, at a smaller computational cost.

A number of estimation algorithms have been proposed specifically for the C-SLAM problem, as alternatives to the EKF estimator [8]. In [9], a Set Membership (SM) technique is developed based on the premise that all sensor errors are bounded. This assumption allows for the definition of the set of feasible state vectors, which is propagated through time using Linear Programming and set approximation methods. SM provides guaranteed uncertainty regions for all robots and landmarks at each time step. In the work of Thrun [10], an algorithm that integrates Maximum Likelihood (ML) incremental map building with Monte Carlo localization is proposed. The pose of the robots is propagated using a particle filter representation of their belief functions, while the map is compiled in a distributed manner among robots, using laser range data. In [11], the constrained local submap filter is employed, enabling each vehicle to build a map of its surroundings independently from the rest of the team. In this case, a global map is created by periodically fusing the vehicles' submaps. In [12], [13], an elevation map of an outdoor area is created using multiple robots that localize in a common coordinate frame. Finally, in [14] a manifold representation of 2D space, and a ML estimator are employed. This approach offers a method for alleviating the problem of map inconsistency in environments containing loops, at the expense of increased complexity.

Our work does not address the aforementioned implementation issues of C-SLAM. We assume perfect data association and seek to characterize the theoretically attainable estimation accuracy by providing bounds for the steady state covariance of the position estimates. To the best of our knowledge, the properties of the estimates' covariance matrix in C-SLAM are only studied in the work of Fenwick et al. [4], [8]. Linear timeinvariant models for both the propagation and measurement equations are employed, and it is shown that the determinant of any principal submatrix of the map's covariance matrix decreases monotonically as successive observations are made.
Under the additional assumption that the robots receive noisefree odometry measurements, it is proven that at steady state, all of the vehicle and feature position estimates become fully correlated and lower bounds for the covariance of all vehicles and features are derived. However, the proposed lower bounds cannot be employed for determining the performance of CSLAM in the case of robots in motion exploring an unknown area. In such a scenario, the global coordinate frame can be arbitrarily defined, thus at least one of the robots has perfect knowledge of its initial position, and the described lower bound reduces to zero.

The main contribution of the work presented in this paper is the characterization of the steady-state accuracy of the position estimates in C-SLAM. This is achieved by deriving analytical upper bounds for the worst-case value as well as for the expected value of the steady-state covariance matrix of the position estimates. What distinguishes these results from previous ones is that the analysis is based on the actual (non-linear) system and measurement equations for robots navigating in 2D. Besides the naturally arising assumption of connectedness, no additional assumptions on the structure of the RPMG are imposed. Furthermore, the analysis encompasses the case of a heterogeneous (in terms of sensor accuracy) group of robots and is thus applicable to the study of a broad class of applications.

\section{Problem Formulation}

Consider a group of $M$ mobile robots, denoted as $r_{1}$, $r_{2}, \ldots, r_{M}$, moving on a planar surface, in an environment that contains $N$ landmarks, denoted as $L_{1}, L_{2}, \ldots, L_{N}$. The robots use proprioceptive measurements (e.g., from odometric or inertial sensors) to propagate their state (position) estimates, and are equipped with exteroceptive sensors (e.g., laser range finders) that enable them to measure the relative position of other robots and landmarks. All the measurements are fused using an Extended Kalman Filter (EKF) in order to produce estimates of the position of the robots and the landmarks. In our formulation, it is assumed that an upper bound for the variance of the errors in the robots' orientation estimates can be determined a priori. This allows us to decouple the task of position estimation from that of orientation estimation and facilitates the derivation of an analytical upper bound on the positioning uncertainty.

The robots' orientation uncertainty is bounded when, for example, absolute orientation measurements from a compass or sun sensor are available, or when the perpendicularity of the walls in an indoor environment is used to infer orientation. In cases where neither approach is possible, our analysis still holds under the condition that a conservative upper bound for the orientation uncertainty of each robot is determined by alternative means, e.g., by estimating the maximum orientation error accumulated, over a certain period of time, due to the integration of noise in the odometric measurements [15]. It should be noted that the requirement for bounded orientation error covariance is not too restrictive: In the EKF framework, the nonlinear state propagation and measurement equations 
are linearized around the estimates of the robots' orientation. If the errors in these estimates are allowed to increase unbounded, the linearization will unavoidably become erroneous and the estimates will diverge. Furthermore, large errors in the estimates for the robots' orientation in SLAM result in erroneous data association, that may have detrimental effects on the filter stability. Thus, in the vast majority of practical situations, provisions are made in order to constrain the robots' orientation uncertainty within given limits.

In our formulation, the metric employed for describing the accuracy of position estimation in C-SLAM is the covariance matrix of the position estimates. It is well known that the time evolution of the covariance matrix in the EKF framework is described by the propagation and update equations (cf. Eqs. (5) and (16)). Combining these equations yields the Riccati recursion (cf. Eq. (17)), whose solution is the covariance of the error in the state estimate at each time step, right after the propagation phase of the EKF. In the case of C-SLAM, the matrix coefficients in this recursion are time-varying and a general closed-form expression for the time evolution of the covariance matrix does not exist. We thus resort to deriving upper bounds for the covariance, by exploiting the convexity and monotonicity properties of the Riccati recursion (cf. Lemmas 4.1 and 4.4). These properties allow for the formulation of constant coefficient Riccati recursions, whose solutions provide upper bounds for the positioning uncertainty in C-SLAM.

\section{A. Position propagation}

The discrete-time kinematic equations for the $i$-th robot are

$$
\begin{aligned}
& x_{r_{i}}(k+1)=x_{r_{i}}(k)+V_{i}(k) \delta t \cos \left(\phi_{i}(k)\right) \\
& y_{r_{i}}(k+1)=y_{r_{i}}(k)+V_{i}(k) \delta t \sin \left(\phi_{i}(k)\right)
\end{aligned}
$$

where $V_{i}(k)$ denotes the robot's translational velocity at time $k$ and $\delta t$ is the sampling period. In the Kalman filter framework, the estimates of the robot's position are propagated using the measurements of the robot's velocity, $V_{m_{i}}(k)$, and the estimates of the robot's orientation, $\hat{\phi}_{i}(k)$. By linearizing Eqs. (1) and (2), the error propagation equation for the robot's position is derived:

$$
\begin{aligned}
& {\left[\begin{array}{l}
\widetilde{x}_{r_{i_{k+1} \mid k}} \\
\widetilde{y}_{r_{i_{k+1} \mid k}}
\end{array}\right]=\left[\begin{array}{cc}
1 & 0 \\
0 & 1
\end{array}\right]\left[\begin{array}{c}
\widetilde{x}_{r_{i_{k \mid k}}} \\
\widetilde{y}_{r_{i_{k} \mid k}}
\end{array}\right]} \\
& +\left[\begin{array}{cc}
\delta t \cos \left(\hat{\phi}_{i}(k)\right) & -V_{m_{i}}(k) \delta t \sin \left(\hat{\phi}_{i}(k)\right) \\
\delta t \sin \left(\hat{\phi}_{i}(k)\right) & V_{m_{i}}(k) \delta t \cos \left(\hat{\phi}_{i}(k)\right)
\end{array}\right]\left[\begin{array}{c}
w_{V_{i}}(k) \\
\widetilde{\phi}_{i}(k)
\end{array}\right] \\
& \Leftrightarrow \widetilde{X}_{r_{i_{k+1} \mid k}}=I_{2 \times 2} \widetilde{X}_{r_{i_{k \mid k}}}+G_{r_{i}}(k) W_{i}(k), \quad i=1 \ldots M
\end{aligned}
$$

where $^{1} w_{V_{i}}(k)$ is a zero-mean white Gaussian noise sequence of variance $\sigma_{V_{i}}^{2}$, affecting the velocity measurements and $\widetilde{\phi}_{i}(k)$ is the error in the robot's orientation estimate at time $k$. This is modeled as a zero-mean white Gaussian noise sequence of variance $\sigma_{\phi_{i}}^{2}$.

\footnotetext{
${ }^{1}$ Throughout this paper, $\mathbf{0}_{m \times n}$ denotes the $m \times n$ matrix of zeros, $\mathbf{1}_{m \times n}$ denotes the $m \times n$ matrix of ones, and $I_{n \times n}$ denotes the $n \times n$ identity matrix.
}

From Eq. (3), we deduce that the covariance matrix of the system noise affecting the $i$-th robot is:

$$
\begin{aligned}
Q_{r_{i}}(k) & =E\left\{G_{r_{i}}(k) W_{i}(k) W_{i}^{T}(k) G_{r_{i}}^{T}(k)\right\} \\
& =C\left(\hat{\phi}_{i}(k)\right)\left[\begin{array}{cc}
\delta t^{2} \sigma_{V_{i}}^{2} & 0 \\
0 & \delta t^{2} V_{m_{i}}^{2}(k) \sigma_{\phi_{i}}^{2}
\end{array}\right] C^{T}\left(\hat{\phi}_{i}(k)\right)
\end{aligned}
$$

where $C\left(\hat{\phi}_{i}\right)$ denotes the $2 \times 2$ rotation matrix associated with $\hat{\phi}_{i}$. The landmarks are modeled as static points in 2D space, and therefore the state propagation equations are

$$
X_{L_{i}}(k+1)=X_{L_{i}}(k), \text { for } i=1 \ldots N
$$

Similarly, the estimates for the landmark positions are propagated using the relations $\hat{X}_{L_{i} k+1 \mid k}=\hat{X}_{L_{i} k \mid k}$, for $i=$ $1 \ldots N$ while the errors are propagated by $\widetilde{X}_{L_{i} k+1 \mid k}=$ $\widetilde{X}_{L_{i} k \mid k}$, for $i=1 \ldots N$. The state vector for the entire system is defined as the stacked vector comprising of the positions of the $M$ robots and $N$ landmarks, i.e.,

$$
X=\left[\begin{array}{llllll}
X_{r_{1}}^{T} & \cdots & X_{r_{M}}^{T} & X_{L_{1}}^{T} & \cdots & X_{L_{N}}^{T}
\end{array}\right]^{T}
$$

Hence, the state transition matrix for the entire system at time step $k$ is $\boldsymbol{\Phi}_{k}=I_{(2 M+2 N) \times(2 M+2 N)}$ and the covariance matrix of the system noise is:

$\mathbf{Q}(k)=\left[\begin{array}{cccc}Q_{r_{1}}(k) & \cdots & \mathbf{0}_{2 \times 2} & \\ \vdots & \ddots & \vdots & \mathbf{0}_{2 M \times 2 N} \\ \mathbf{0}_{2 \times 2} & \cdots & Q_{r_{M}}(k) & \\ & \mathbf{0}_{2 N \times 2 M} & \mathbf{0}_{2 N \times 2 N}\end{array}\right]=\mathbf{G Q}_{r}(k) \mathbf{G}^{T}$

where $\mathbf{Q}_{r}(k)=\operatorname{Diag}\left(Q_{r_{i}}(k)\right)$, with $\operatorname{Diag}(\cdot)$ denoting a block diagonal matrix, and

$$
\mathbf{G}=\left[\begin{array}{l}
I_{2 M \times 2 M} \\
\mathbf{0}_{2 N \times 2 M}
\end{array}\right]
$$

The equation for propagating the covariance matrix of the state error is written as:

$$
\mathbf{P}_{k+1 \mid k}=\mathbf{P}_{k \mid k}+\mathbf{Q}(k)=\mathbf{P}_{k \mid k}+\mathbf{G Q}_{r}(k) \mathbf{G}^{T}
$$

where $\mathbf{P}_{k+1 \mid k}=E\left\{\tilde{X}_{k+1 \mid k} \tilde{X}_{k+1 \mid k}^{T}\right\}$ and $\mathbf{P}_{k \mid k}=$ $E\left\{\widetilde{X}_{k \mid k} \widetilde{X}_{k \mid k}^{T}\right\}$ are the covariance of the error in the estimate of $X(k+1)$ and $X(k)$ respectively, after measurements up to time $k$ have been processed.

\section{B. Measurement Model}

At every time step, the robots perform robot-to-robot and robot-to-landmark relative position measurements. The relative position measurement between robots $r_{i}$ and $r_{m}$ is given by:

$$
z_{r_{i} r_{m}}=C^{T}\left(\phi_{i}\right)\left(X_{r_{m}}-X_{r_{i}}\right)+n_{z_{r_{i} r_{m}}}
$$

where $r_{i}\left(r_{m}\right)$ is the observing (observed) robot, and $n_{z_{r_{i} r_{m}}}$ is the noise affecting this measurement. Similarly, the measurement of the relative position between $r_{i}$ and $L_{n}$ is given by:

$$
z_{r_{i} L_{n}}=C^{T}\left(\phi_{i}\right)\left(X_{L_{n}}-X_{r_{i}}\right)+n_{z_{r_{i} L_{n}}}
$$


The similarity of the preceding two measurement equations allows us to treat both types of measurements in a uniform manner. We denote by $T_{i j}$ the target of the $j$-th measurement performed by robot $i$, i.e.,

$$
T_{i j} \in\left\{r_{1}, r_{2}, \cdots, r_{M}, L_{1}, L_{2}, \cdots, L_{N}\right\} \backslash\left\{r_{i}\right\}
$$

Thus, the general form of the relative position measurement equation is:

$$
z_{i j}=C^{T}\left(\phi_{i}\right)\left(X_{T_{i j}}-X_{r_{i}}\right)+n_{z_{i j}}
$$

Assuming that the $i$-th robot performs $M_{i}$ relative position measurements, the index $j$ assumes integer values in the range $\left[1, M_{i}\right]$ to describe these measurements. By linearizing the last expression, the measurement error equation is obtained:

$$
\begin{aligned}
\widetilde{z}_{i j}(k+1) & =z_{i j}(k+1)-\hat{z}_{i j}(k+1) \\
& =H_{i j}(k+1) \widetilde{X}_{k+1 \mid k}+\Gamma_{i j}(k+1) n_{i j}(k+1)
\end{aligned}
$$

where

$$
\begin{aligned}
& H_{i j}(k+1)=C^{T}\left(\hat{\phi}_{i}(k+1)\right) H_{o_{i j}} \\
& H_{o_{i j}}=\left[\begin{array}{lllllll}
0_{2 \times 2} & \cdots & \underbrace{-I_{2 \times 2}}_{r_{i}} & \cdots & \underbrace{I_{2 \times 2}}_{T_{i j}} & \cdots & 0_{2 \times 2}
\end{array}\right] \\
& \widetilde{X}_{k+1 \mid k}=\left[\begin{array}{lllll}
\cdots & \widetilde{X}_{r_{i}}^{T} & \cdots & \tilde{X}_{T_{i j}}^{T} & \cdots
\end{array}\right]_{k+1 \mid k}^{T} \\
& \Gamma_{i j}(k)=\left[\begin{array}{ll}
I_{2 \times 2} & -C^{T}\left(\hat{\phi}_{i}(k+1)\right) J \widehat{\Delta p}_{i j}(k+1)
\end{array}\right] \\
& J=\left[\begin{array}{cc}
0 & -1 \\
1 & 0
\end{array}\right], \quad n_{i j}(k)=\left[\begin{array}{c}
n_{z_{i j}}(k) \\
\widetilde{\phi}_{i}(k)
\end{array}\right] \\
& \widehat{\Delta p}_{i j}(k+1)=\widehat{X}_{T_{i j k+1 \mid k}}-\widehat{X}_{r_{i k+1 \mid k}}
\end{aligned}
$$

The covariance of the error in this measurement is given by

$$
\begin{aligned}
{ }^{i} R_{j j}(k+1) & =\Gamma_{i j}(k+1) E\left\{n_{i j}(k+1) n_{i j}^{T}(k+1)\right\} \Gamma_{i j}^{T}(k+1) \\
& =R_{z_{i j}}(k+1)+R_{\tilde{\phi}_{i j}}(k+1)
\end{aligned}
$$

This expression encapsulates all sources of noise and uncertainty that contribute to the measurement error $\widetilde{z}_{i j}(k+1)$. More specifically, $R_{z_{i j}}(k+1)$ is the covariance component attributed to the measurement noise $n_{z_{i j}}(k+1)$, and $R_{\tilde{\phi}_{i j}}(k+1)$ is the additional covariance term due to the error $\tilde{\phi}_{i}(k+1)$ in the orientation estimate of the measuring robot.

Assuming that each relative position measurement is comprised of a distance measurement $\rho_{i j}$ and a bearing measurement $\theta_{i j}$, affected by two independent white zero-mean Gaussian noise sequences $n_{\rho_{i j}}$ and $n_{\theta_{i j}}$ respectively, the term $R_{z_{i j}}(k+1)$ can be expressed as [16]:

$$
\begin{aligned}
& R_{z_{i j}}(k+1)=E\left\{n_{z_{i j}}(k+1) n_{z_{i j}}^{T}(k+1)\right\} \\
& \quad=C^{T}\left(\hat{\phi}_{i}\right)\left(\frac{\sigma_{\rho_{i}}^{2}}{\hat{\rho}_{i j}^{2}} \widehat{\Delta p}_{i j} \widehat{\Delta p}_{i j}^{T}+\sigma_{\theta_{i}}^{2} J \widehat{\Delta p_{i j}} \widehat{\Delta p}_{i j}^{T} J^{T}\right) C\left(\hat{\phi}_{i}\right)
\end{aligned}
$$

where time indices have been dropped for simplicity. The variances of the noise in the distance and bearing measurements are denoted as $\sigma_{\rho_{i}}^{2}=E\left\{n_{\rho_{i}}^{2}\right\}$ and $\sigma_{\theta_{i}}^{2}=E\left\{n_{\theta_{i}}^{2}\right\}$ respectively.

The error in the orientation estimate of the measuring robot introduces an additional error component to all relative position measurements recorded by this robot, and renders them correlated. As shown in [16], the additional covariance term for each measurement is equal to:

$$
\begin{aligned}
R_{\tilde{\phi}_{i j}}(k+1) & =C^{T}\left(\hat{\phi}_{i}\right) J \widehat{\Delta p}_{i j} E\left\{\tilde{\phi}_{i}^{2}\right\} \widehat{\Delta p}_{i j}^{T} J^{T} C\left(\hat{\phi}_{i}\right) \\
& =\sigma_{\phi_{i}}^{2} C^{T}\left(\hat{\phi}_{i}\right) J \widehat{\Delta p}_{i j} \widehat{\Delta p}_{i j}^{T} J^{T} C\left(\hat{\phi}_{i}\right)
\end{aligned}
$$

while the matrix of correlation between the errors in the measurements $z_{i j}(k+1)$ and $z_{i \ell}(k+1)$ is:

$$
\begin{aligned}
{ }^{i} R_{j \ell}(k+1) & =\Gamma_{i j}(k) E\left\{n_{i j}(k+1) n_{i \ell}^{T}(k+1)\right\} \Gamma_{i \ell}^{T}(k) \\
& =\sigma_{\phi_{i}}^{2} C^{T}\left(\hat{\phi}_{i}\right) J \widehat{\Delta p_{i j}} \widehat{\Delta p}_{i \ell}^{T} J^{T} C\left(\hat{\phi}_{i}\right)
\end{aligned}
$$

Since the $i$-th robot performs $M_{i}$ relative position measurements at each time step, the covariance matrix of these measurements, $\boldsymbol{R}_{i}(k+1)$, is defined as a block matrix whose $\ell m$-th $2 \times 2$ submatrix is ${ }^{i} R_{\ell m}(k+1)$, for $\ell, m=1 \ldots M_{i}$, defined in Eqs. (10)-(12). Substitution from these equations and simple algebraic manipulation yields

$$
\boldsymbol{R}_{i}(k+1)=\boldsymbol{\Xi}_{\hat{\phi}_{i}}^{T} \boldsymbol{R}_{o_{i}}(k+1) \boldsymbol{\Xi}_{\hat{\phi}_{i}}
$$

where $\boldsymbol{\Xi}_{\hat{\phi}_{i}}=I_{M_{i} \times M_{i}} \otimes C\left(\hat{\phi}_{i}\right)$, with $\otimes$ denoting the Kronecker matrix product, and

$$
\begin{aligned}
\boldsymbol{R}_{o_{i}}(k+1)= & \sigma_{\rho_{i}}^{2} I_{2 M_{i} \times 2 M_{i}}-D_{i} \operatorname{diag}\left(\frac{\sigma_{\rho_{i}}^{2}}{\hat{\rho}_{i j}^{2}}\right) D_{i}^{T} \\
& +\sigma_{\theta_{i}}^{2} D_{i} D_{i}^{T}+\sigma_{\phi_{i}}^{2} D_{i} \mathbf{1}_{M_{i} \times M_{i}} D_{i}^{T}
\end{aligned}
$$

In this last expression, $D_{i}=\operatorname{Diag}\left(J \widehat{\Delta p}_{i j}\right)$ is the block diagonal matrix with diagonal elements $J \widehat{\Delta p}_{i j}, j=1 \ldots M_{i}$.

The measurement matrix describing the relative position measurements performed by robot $i$ at each time step is a matrix whose block rows are $H_{i j}, j=1 \ldots M_{i}$, i.e.:

$$
\boldsymbol{H}_{i}(k+1)=\boldsymbol{\Xi}_{\hat{\phi}_{i}}^{T} \boldsymbol{H}_{o_{i}}
$$

where $\boldsymbol{H}_{o_{i}}$ is a constant matrix with block rows $H_{o_{i j}}, j=$ $1 \ldots M_{i}$ (cf. Eq. (9)).

The measurement matrix for the entire system, $\mathbf{H}(k+1)$, is defined as the block matrix with block rows $\boldsymbol{H}_{i}(k+1)$. Since the measurements performed by different robots are independent, the associated covariance matrix, $\mathbf{R}(k+1)$, is a block diagonal matrix with elements $\boldsymbol{R}_{i}(k+1)$. The covariance update equation of the EKF is written as

$$
\mathbf{P}_{k+1 \mid k+1}=\mathbf{P}_{k+1 \mid k}-\mathbf{P}_{k+1 \mid k} \mathbf{H}^{T}(k+1) \mathbf{S}^{-1} \mathbf{H}(k+1) \mathbf{P}_{k+1 \mid k}
$$

with $\mathbf{S}=\mathbf{H}(k+1) \mathbf{P}_{k+1 \mid k} \mathbf{H}^{T}(k+1)+\mathbf{R}(k+1)$. Substitution from Eqs. (13) and (15) and simple algebraic manipulation leads to the orientation-dependent terms being cancelled out, and yields the expression

$$
\mathbf{P}_{k+1 \mid k+1}=\mathbf{P}_{k+1 \mid k}-\mathbf{P}_{k+1 \mid k} \mathbf{H}_{o}^{T} \mathbf{S}_{o}^{-1} \mathbf{H}_{o} \mathbf{P}_{k+1 \mid k}
$$

with $\mathbf{S}_{o}=\mathbf{H}_{o} \mathbf{P}_{k+1 \mid k} \mathbf{H}_{o}^{T}+\mathbf{R}_{o}(k+1)$. In these equations $\mathbf{H}_{o}$ is a matrix whose block rows are $\boldsymbol{H}_{o_{i}}$ while $\mathbf{R}_{o}$ is a block diagonal matrix with elements $\boldsymbol{R}_{o_{i}}$. 


\section{SLAM Positioning ACCURACy Characterization}

The time evolution of the covariance matrix of the position estimates in C-SLAM is described by the Riccati recursion, which can be derived by substituting the expression from Eq. (16) into Eq. (5). The resulting expression is:

$$
\begin{aligned}
\mathbf{P}_{k+1} & =\mathbf{P}_{k}-\mathbf{P}_{k} \mathbf{H}_{o}^{T}\left(\mathbf{H}_{o} \mathbf{P}_{k} \mathbf{H}_{o}^{T}+\mathbf{R}_{o}(k+1)\right)^{-1} \mathbf{H}_{o} \mathbf{P}_{k} \\
& +\mathbf{G Q}_{r}(k+1) \mathbf{G}^{T}
\end{aligned}
$$

where we use the substitutions $\mathbf{P}_{k}=\mathbf{P}_{k+1 \mid k}$ and $\mathbf{P}_{k+1}=$ $\mathbf{P}_{k+2 \mid k+1}$ to simplify the notation. We note that the matrices $\mathbf{Q}_{r}(k+1)$ and $\mathbf{R}_{o}(k+1)$ in this Riccati recursion are time varying, and thus a closed form expression for $\mathbf{P}$ cannot be derived for the general case. However, by exploiting the monotonicity and concavity properties of the Riccati recursion, we are able to derive upper bounds for the worst-case value, as well as for the average value of the covariance matrix at steady state. At this point we note that in the ensuing derivations, the initial covariance matrix of the position estimates is assumed to be equal to

$$
\mathbf{P}_{0}=\left[\begin{array}{cc}
\mathbf{P}_{r r_{0}} & \mathbf{0}_{2 M \times 2 N} \\
\mathbf{0}_{2 N \times 2 M} & \mathbf{P}_{L L_{0}}
\end{array}\right]
$$

i.e., we assume that the position estimates for the robots and the map features are initially uncorrelated, which is the case at the onset of a mapping task within an unknown area.

\section{A. Bound on Worst-Case Steady State Covariance}

In this section, we derive an upper bound for the steady state covariance matrix in C-SLAM. It can be shown that the right-hand side of Eq. (17) is a matrix-increasing function of the covariance matrices $\mathbf{Q}(k+1)$ and $\mathbf{R}_{o}(k+1)$, as well as of the state covariance matrix $\mathbf{P}_{k}$. These properties allow us to prove the following lemma [16]:

Lemma 4.1: If $\mathbf{R}_{u}$ and $\mathbf{Q}_{r_{u}}$ are matrices such that $\mathbf{R}_{u} \succeq$ $\mathbf{R}_{o}(k)$ and $\mathbf{Q}_{r_{u}} \succeq \mathbf{Q}_{r}(k)$, for all $k \geq 0$, then the solution to the Riccati recursion

$$
\begin{aligned}
\mathbf{P}_{k+1}^{u} & =\mathbf{P}_{k}^{u}-\mathbf{P}_{k}^{u} \mathbf{H}_{o}^{T}\left(\mathbf{H}_{o} \mathbf{P}_{k}^{u} \mathbf{H}_{o}^{T}+\mathbf{R}_{u}\right)^{-1} \mathbf{H}_{o} \mathbf{P}_{k}^{u} \\
& +\mathbf{G Q}_{r_{u}} \mathbf{G}^{T}
\end{aligned}
$$

with the initial condition $\mathbf{P}_{0}^{u}=\mathbf{P}_{0}$, satisfies $\mathbf{P}_{k}^{u} \succeq \mathbf{P}_{k}$ for all $k \geq 0$.

In order to derive an upper bound for $\mathbf{Q}_{r}(k)$, we note that since $C\left(\hat{\phi}_{i}\right)$ is an orthonormal matrix, the eigenvalues of $Q_{r_{i}}(k)$ are equal to $\delta t^{2} \sigma_{V_{i}}^{2}$ and $\delta t^{2} V_{m_{i}}^{2}(k) \sigma_{\phi_{i}}^{2}$ (cf. Eq. (4)). Assuming that the velocity of each robot is approximately constant and equal to $V_{i}$, we denote

$q_{i}=\max \left(\delta t^{2} \sigma_{V_{i}}^{2}, \delta t^{2} V_{m_{i}}^{2}(k) \sigma_{\phi_{i}}^{2}\right) \simeq \max \left(\delta t^{2} \sigma_{V_{i}}^{2}, \delta t^{2} V_{i}^{2} \sigma_{\phi_{i}}^{2}\right)$

This definition states that $q_{i}$ is the largest eigenvalue of $Q_{r_{i}}(k+1)$, and therefore

$$
Q_{r_{i}}(k) \preceq q_{i} I_{2 \times 2} \Rightarrow \mathbf{Q}_{\mathbf{r}}(k) \preceq \operatorname{Diag}\left(q_{i} I_{2 \times 2}\right)=\mathbf{Q}_{r_{u}}
$$

An upper bound for $\mathbf{R}_{o}(k+1)$ can be derived by considering the maximum distance, $\rho_{o_{i}}$, at which relative position measurements can be recorded by robot $i$. This distance can, for example, be determined by the maximum range of the robots' relative position sensors, or, by the size of the area to be mapped. It can be shown that [16]

$\boldsymbol{R}_{i}(k+1) \preceq\left(\sigma_{\rho_{i}}^{2}+M_{i} \sigma_{\phi_{i}}^{2} \rho_{o}^{2}+\sigma_{\theta_{i}}^{2} \rho_{o}^{2}\right) I_{2 M_{i} \times 2 M_{i}}=r_{i} I_{2 M_{i} \times 2 M_{i}}$ and thus an upper bound on $\mathbf{R}_{o}(k+1)$ is computed as

$$
\mathbf{R}_{o}(k+1) \preceq \operatorname{Diag}\left(r_{i} I_{2 M_{i} \times 2 M_{i}}\right)=\mathbf{R}_{u}
$$

Having derived upper bounds for $\mathbf{Q}_{r}(k)$ and $\mathbf{R}_{o}(k+1)$, mere substitution in Eq. (19) and numerical evaluation of the solution to the resulting recursion, yields an upper bound on the maximum possible uncertainty of the position estimates in C-SLAM, at any time instant after the deployment of the robot team. However, for many applications it is important to evaluate the performance of SLAM at steady state, i.e., when the covariance of the map has converged to a constant value ([4]). To this end, it is necessary to evaluate the solution to the recursion in Eq. (19) after sufficient time, i.e., as $k \rightarrow \infty$. This computation is simplified by employing the following Lemma, adapted from [17]:

Lemma 4.2: Suppose $\mathbf{P}_{k}^{u(0)}$ is the solution to the discrete time Riccati recursion in Eq. (19) with initial value $\mathbf{P}_{0}^{u}=0$. Then the solution to the same Riccati recursion but with an arbitrary initial condition $\mathbf{P}_{0}$ is given by the identity

$\mathbf{P}_{k+1}^{u}-\mathbf{P}_{k+1}^{u(0)}=\boldsymbol{\Phi}(0, k+1)\left(I_{\xi \times \xi}+\mathbf{P}_{0} \mathbf{J}_{k+1}\right)^{-1} \mathbf{P}_{0} \boldsymbol{\Phi}^{T}(0, k+1)$

where $\xi=2 M+2 N$, and $\boldsymbol{\Phi}(0, k+1)$ is given by

$$
\boldsymbol{\Phi}(0, k+1)=\left(I_{\xi \times \xi}-\mathbf{K}_{p} \mathbf{H}_{o}\right)^{k+1}\left(I_{\xi \times \xi}+\mathbf{P} \mathbf{J}_{k+1}\right)
$$

In these expressions, $\mathbf{P}$ is any solution to the Discrete Algebraic Riccati Equation (DARE):

$$
\mathbf{P}=\mathbf{P}+\mathbf{G Q}_{r_{u}} \mathbf{G}^{T}-\mathbf{P} \mathbf{H}_{o}^{T}\left(\mathbf{H}_{o} \mathbf{P} \mathbf{H}_{o}^{T}+\mathbf{R}_{u}\right)^{-1} \mathbf{H}_{o} \mathbf{P},
$$

and $\mathbf{K}_{p}=\mathbf{P} \mathbf{H}_{o}^{T}\left(\mathbf{R}_{u}+\mathbf{H}_{o} \mathbf{P} \mathbf{H}_{o}^{T}\right)^{-1} . \mathbf{J}_{k}$ denotes the solution to the dual Riccati recursion:

$\mathbf{J}_{k+1}=\mathbf{J}_{k}-\mathbf{J}_{k} \mathbf{G}\left(\mathbf{Q}_{r_{u}}^{-1}+\mathbf{G}^{T} \mathbf{J}_{k} \mathbf{G}\right)^{-1} \mathbf{G}^{T} \mathbf{J}_{k}+\mathbf{H}_{o}^{T} \mathbf{R}_{u}^{-1} \mathbf{H}_{o}$

with zero initial condition, $\mathbf{J}_{0}=0$.

Lemma 4.2 simplifies the evaluation of the steady-state value of $\mathbf{P}_{k}^{u}$, since the solution to the Riccati recursion with zero initial condition is easily derived. We note that the Riccati recursion in Eq. (19) describes the time evolution of the covariance for a deduced C-SLAM scenario, in which both the robots' kinematic equations and the measurement equations are time invariant. Zero initial covariance of the landmarks' position estimates, corresponds to a perfectly known map. In this case the robots essentially perform cooperative localization, while the robot-to-landmark measurements are equivalent to absolute position measurements. Thus, the resulting system 
is observable and the steady-state solution to the Riccati equation is:

$$
\mathbf{P}_{\infty}^{u(0)}=\left[\begin{array}{cc}
\mathbf{P}_{r r_{\infty}}^{u} & \mathbf{0}_{2 M \times 2 N} \\
\mathbf{0}_{2 N \times 2 M} & \mathbf{0}_{2 N \times 2 N}
\end{array}\right]
$$

with

$$
\mathbf{P}_{r r_{\infty}}^{u}=\mathbf{Q}_{r_{u}}^{1 / 2} \mathbf{U} \operatorname{diag}\left(\frac{1}{2}+\sqrt{\frac{1}{4}+\frac{1}{\lambda_{i}}}\right) \mathbf{U}^{T} \mathbf{Q}_{r_{u}}^{1 / 2}
$$

In the last expression the quantities $\mathbf{U}$ and $\lambda_{i}, i=1 \ldots 2 M$ are defined as the matrix of eigenvectors and the eigenvalues of $\mathbf{C}=\mathbf{Q}_{r_{u}}^{1 / 2} \mathbf{I}_{r} \mathbf{Q}_{r_{u}}^{1 / 2}$ respectively, where

$$
\mathbf{I}_{r}=\left[\begin{array}{ll}
I_{2 M \times 2 M} & \mathbf{0}_{2 M \times 2 N}
\end{array}\right] \mathbf{H}_{o}^{T} \mathbf{R}_{u}^{-1} \mathbf{H}_{o}\left[\begin{array}{l}
I_{2 M \times 2 M} \\
\mathbf{0}_{2 N \times 2 M}
\end{array}\right]
$$

It is interesting to note that $\mathbf{H}_{o}^{T} \mathbf{R}_{u}^{-1} \mathbf{H}_{o}$ represents the information matrix associated with the measurements in the deduced linear time-invariant system and $\mathbf{I}_{r}$ is the submatrix expressing the information about the robots' positions.

The rest of the derivations for the upper bound on the steady state covariance matrix involve only algebraic manipulations, and are not included here due to space limitations. The interested reader is referred to [16] for the details of the intermediate steps. The final result is stated in the following lemma:

Lemma 4.3: The worst-case covariance matrix in C-SLAM is bounded above by the matrix

$$
\mathbf{P}_{\infty}^{u}=\mathbf{P}_{\infty}^{u(0)}+\mathbf{1}_{(M+N) \times(M+N)} \otimes \Theta^{-1}
$$

where $\mathbf{P}_{\infty}^{u(0)}$ is defined in Eq. (22) and

$$
\begin{aligned}
\Theta & =\left(\mathbf{1}_{1 \times N} \otimes I_{2 \times 2}\right) \mathbf{P}_{L L_{0}}^{-1}\left(\mathbf{1}_{N \times 1} \otimes I_{2 \times 2}\right) \\
& +\left(\mathbf{1}_{1 \times M} \otimes I_{2 \times 2}\right)\left(\mathbf{J}_{r r_{\infty}}^{-1}+\mathbf{P}_{r r_{0}}\right)^{-1}\left(\mathbf{1}_{M \times 1} \otimes I_{2 \times 2}\right)
\end{aligned}
$$

with

$$
\mathbf{J}_{r r_{\infty}}=\mathbf{Q}_{r_{u}}^{-1 / 2} \mathbf{U} \operatorname{diag}\left(\frac{\lambda_{i}}{2}+\sqrt{\frac{\lambda_{i}^{2}}{4}+\lambda_{i}}\right) \mathbf{U}^{T} \mathbf{Q}_{r_{u}}^{-1 / 2}
$$

Note that the first term in Eq. (23) depends only on the RPMG and the accuracy of the robots' sensors, while the second term encapsulates the effect of the initial uncertainty.

A case of particular interest in C-SLAM is that of a robot team building a map of an area for which no prior knowledge exists. We model this scenario by setting $\mathbf{P}_{L L_{0}}=\mu I$, with $\mu \rightarrow \infty$, which yields the following simplified expression for matrix $\Theta$ :

$$
\Theta=\left(\mathbf{1}_{1 \times M} \otimes I_{2 \times 2}\right)\left(\mathbf{J}_{r r_{\infty}}^{-1}+\mathbf{P}_{r r_{0}}\right)^{-1}\left(\mathbf{1}_{M \times 1} \otimes I_{2 \times 2}\right)
$$

\section{$B$. Bound on the average steady state covariance}

The expression in Eq. (23) provides an upper bound on the covariance matrix of C-SLAM for a robot team with a given set of sensors and a known RPMG. This bound holds under any possible configuration of the landmarks in space, and regardless of the trajectories of the robots within the area. However, when considering the type of features of the environment to be treated as landmarks (e.g., visual features, prominent geometric features), it is beneficial to select them so that they are abundant in the environment and evenly distributed throughout it. This way, a more detailed and accurate map of an area can be created. In such cases, the density of landmarks in the environment can be modeled $a$ priori, for example, by a uniform probability density function (pdf). Knowledge of the distribution of the relative positions between the robots and landmarks allows us to compute the average value of the matrix $\mathbf{R}_{o}(k+1)$. This information can be exploited in order to compute a tighter upper bound for the expected steady state covariance of the position estimates.

Specifically, it can be shown that the right hand side of Eq. (17) is a concave function of the matrices $\mathbf{P}_{k}$ and $\mathbf{R}_{o}(k+1)$. This property enables us to employ Jensen's inequality ([18]) to prove, by induction, the following lemma [16]:

Lemma 4.4: If $\overline{\mathbf{R}}=E\left\{\mathbf{R}_{o}(k)\right\}$ and $\overline{\mathbf{Q}}_{r}=E\left\{\mathbf{Q}_{r}(k)\right\}$ then the solution to the following Riccati recursion

$$
\begin{aligned}
\overline{\mathbf{P}}_{k+1} & =\overline{\mathbf{P}}_{k}-\overline{\mathbf{P}}_{k} \mathbf{H}_{o}^{T}\left(\mathbf{H}_{o} \overline{\mathbf{P}}_{k} \mathbf{H}_{o}^{T}+\overline{\mathbf{R}}\right)^{-1} \mathbf{H}_{o} \overline{\mathbf{P}}_{k} \\
& +\mathbf{G} \overline{\mathbf{Q}}_{r} \mathbf{G}^{T}
\end{aligned}
$$

with initial condition $\overline{\mathbf{P}}_{0}=\mathbf{P}_{0}$, satisfies $\overline{\mathbf{P}}_{k} \succeq E\left\{\mathbf{P}_{k}\right\}$ for all $k \geq 0$.

The average value of the system noise covariance matrix is easily computed by averaging over all values of orientation of the robots:

$$
E\left\{Q_{r_{i}}\right\}=\frac{\delta t^{2} \sigma_{V_{i}}^{2}+\delta t^{2} V_{i}^{2} \sigma_{\phi_{i}}^{2}}{2} I_{2 \times 2}=\bar{q}_{i} I_{2 \times 2}
$$

and thus

$$
\overline{\mathbf{Q}}_{r}=E\left\{\mathbf{Q}_{r}(k+1)\right\}=\operatorname{Diag}\left(\bar{q}_{i} I_{2 \times 2}\right)
$$

In order to evaluate the expected value of $\mathbf{R}_{o}(k+1)$, we assume a uniform distribution for the positions of the robots and landmarks in a rectangular area of side $\alpha$. Using the definition of $\boldsymbol{R}_{o_{i}}(k+1)$ in Eq. (14), it can be shown that [16]

$$
\begin{aligned}
\overline{\mathbf{R}}_{i} & =E\left\{\boldsymbol{R}_{o_{i}}\right\}=\left(\sigma_{\rho_{i}}^{2} \frac{\alpha^{2}}{2}+\sigma_{\theta_{i}}^{2} \frac{\alpha^{2}}{6}+\sigma_{\phi_{i}}^{2} \frac{\alpha^{2}}{12}\right) I_{2 M_{i} \times 2 M_{i}} \\
& +\sigma_{\phi_{i}}^{2} \frac{\alpha^{2}}{12} \mathbf{1}_{2 M_{i} \times 2 M_{i}}
\end{aligned}
$$

and thus $\overline{\mathbf{R}}=E\left\{\mathbf{R}_{o}\right\}=\operatorname{Diag}\left(\overline{\mathbf{R}}_{i}\right)$. At this point, we note that the uniform distribution employed in the calculation of $\overline{\mathbf{R}}$, was deemed an appropriate model for the positions of the robots and landmarks in the simulation experiments presented in Section V. However, the analysis holds for any pdf. If a different pdf is used, the value of $\overline{\mathbf{R}}$ will not, in general, be given by the preceding expression.

The upper bound for the expected steady-state covariance can be computed by evaluating the solution to the recursion in Eq. (25) after sufficient time. The derivation process followed is analogous to the one presented in the previous section. The 
only difference is that matrices $\overline{\mathbf{R}}$ and $\overline{\mathbf{Q}}_{r}$, instead of $\mathbf{R}_{u}$ and $\mathbf{Q}_{r_{u}}$, respectively, are used. The final result is synopsized in the following lemma:

Lemma 4.5: The expected steady-state covariance of the position estimates in C-SLAM, when the spatial density of landmarks is described by a known pdf, is bounded above by the matrix

$$
\overline{\mathbf{P}}_{\infty}=\left[\begin{array}{cc}
\overline{\mathbf{P}}_{r r_{\infty}} & \mathbf{0}_{2 M \times 2 N} \\
\mathbf{0}_{2 N \times 2 M} & \mathbf{0}_{2 N \times 2 N}
\end{array}\right]+\mathbf{1}_{(M+N) \times(M+N)} \otimes \bar{\Theta}^{-1}
$$

with

and

$$
\overline{\mathbf{P}}_{r r_{\infty}}=\overline{\mathbf{Q}}_{r}^{1 / 2} \overline{\mathbf{U}} \operatorname{diag}\left(\frac{1}{2}+\sqrt{\frac{1}{4}+\frac{1}{\bar{\lambda}_{i}}}\right) \overline{\mathbf{U}}^{T} \overline{\mathbf{Q}}_{r}^{1 / 2}
$$

$$
\begin{aligned}
\bar{\Theta} & =\left(\mathbf{1}_{1 \times N} \otimes I_{2 \times 2}\right) \mathbf{P}_{L L_{0}}^{-1}\left(\mathbf{1}_{N \times 1} \otimes I_{2 \times 2}\right) \\
& +\left(\mathbf{1}_{1 \times M} \otimes I_{2 \times 2}\right)\left(\overline{\mathbf{J}}_{r r_{\infty}}^{-1}+\mathbf{P}_{r r_{0}}\right)^{-1}\left(\mathbf{1}_{M \times 1} \otimes I_{2 \times 2}\right)
\end{aligned}
$$

The quantity $\mathbf{J}_{r r_{\infty}}$ appearing in this last expression is:

$$
\overline{\mathbf{J}}_{r r_{\infty}}=\overline{\mathbf{Q}}_{r}^{-1 / 2} \overline{\mathbf{U}} \operatorname{diag}\left(\frac{\bar{\lambda}_{i}}{2}+\sqrt{\frac{\bar{\lambda}_{i}^{2}}{4}+\bar{\lambda}_{i}}\right) \overline{\mathbf{U}}^{T} \overline{\mathbf{Q}}_{r}^{-1 / 2}
$$

where $\overline{\mathbf{U}}$ and $\bar{\lambda}_{i}, i=1 \ldots 2 M$ are defined based on the singular value decomposition of the matrix $\overline{\mathbf{I}}_{r}$ :

$$
\begin{aligned}
\overline{\mathbf{I}}_{r} & =\left[\begin{array}{ll}
I_{2 M \times 2 M} & \mathbf{0}_{2 M \times 2 N}
\end{array}\right] \mathbf{H}_{o}^{T} \overline{\mathbf{R}}^{-1} \mathbf{H}_{o}\left[\begin{array}{l}
I_{2 M \times 2 M} \\
\mathbf{0}_{2 N \times 2 M}
\end{array}\right] \\
& =\overline{\mathbf{U}} \operatorname{diag}\left(\bar{\lambda}_{i}\right) \overline{\mathbf{U}}^{T}
\end{aligned}
$$

In the special case where the map is initially unknown, matrix $\bar{\Theta}$ assumes the value

$$
\begin{gathered}
\bar{\Theta}=\left(\mathbf{1}_{1 \times M} \otimes I_{2 \times 2}\right)\left(\overline{\mathbf{J}}_{r r_{\infty}}^{-1}+\mathbf{P}_{r r_{0}}\right)^{-1}\left(\mathbf{1}_{M \times 1} \otimes I_{2 \times 2}\right) \\
\text { V. SimULATION RESULTS }
\end{gathered}
$$

A series of experiments in simulation were conducted, in order to validate the preceding theoretical analysis. The simulated robots move in an arena of dimensions $10 \times 10 \mathrm{~m}$, within which point landmarks are located. The velocity of the robots is kept constant at $V=0.25 \mathrm{~m} / \mathrm{s}$, while their orientation changes randomly, using samples drawn from a uniform distribution. To simplify the presentation, a homogeneous robot team is assumed. The standard deviation of the velocity measurement noise is equal to $\sigma_{V}=0.05 \mathrm{~V}$ and the standard deviation of the errors in the orientation estimates is equal to $\sigma_{\phi}=2^{\circ}$, for all robots. Similarly, the values selected for the standard deviations of the exteroceptive measurements of the robots are $\sigma_{\theta}=2^{\circ}$, for the bearing measurements, and $\sigma_{\rho}=0.05 \mathrm{~m}$, for the range measurements. For the results presented in this section, the RPMG shown in Fig. 1(a) is used. It is assumed that initially the robots have perfect knowledge of their positions, while the landmark positions are unknown.

In order to demonstrate the validity of the bound on the worst-case covariance of C-SLAM, provided in Lemma 4.3, a particularly adverse scenario for the placement of the landmarks is considered. Specifically, all the landmarks form a cluster at one corner of the arena, while the robots begin their exploration at the opposite corner (Fig. 1(b)). In this case the exteroceptive measurements provide only a small amount of positioning information during the crucial first few updates. In Fig. 1(c), the time evolution of the covariance of the position estimates for the robots and landmarks is shown and compared to the theoretically-derived steady-state performance bound. Clearly, the upper bound is indeed larger than the steadystate covariance of the landmarks and robots. It is also worth noting that the covariance of the position estimates converges to the same value for all landmarks, while the accuracy of the position estimates varies between robots. These differences result from the non-symmetric topology of the RPMG, which causes each robot to have access to positioning information of different quality.

Although the bound of Lemma 4.3 accounts for the worstcase accuracy of C-SLAM, it does not yield a sufficient performance description when the map features are more evenly distributed in space. In such cases, employing Lemma 4.5 results in a tighter bound on the average positioning uncertainty, as demonstrated in Fig. 2. In this plot, the average values (over 50 runs) of the covariance in C-SLAM, are compared against the theoretically derived bounds on the expected uncertainty. For each run of the algorithm, the locations of the landmarks, as well as the initial positions for the robots, were selected using samples from a uniform distribution. Note that the scale of the axes in Fig. 2 has been changed compared to Fig. 1(c), in order to preserve clarity. Mere comparison of the values for the covariance of the robots' and landmarks' position estimates with the corresponding bounds demonstrates that when available information about the distribution of the landmarks is exploited, i.e., by employing the expressions from Lemma 4.5, a better characterization of the expected accuracy of the position estimates is achieved.

\section{CONCLUSions}

In this paper, we have presented a method for predicting the positioning performance of C-SLAM, without the need to resort to extensive simulations or experimentation. The derived expressions enable us to determine guaranteed steady-state accuracy values for a robot team with a given set of sensors, mapping an area of known size (cf. Lemma 4.3). Moreover, when a model of the distribution of the landmarks in the area is available, application of Lemma 4.5 yields a tight upper bound for the expected value of the steady-state covariance of the position estimates of the robots and landmarks. The aforementioned Lemmas provide functional relations for the positioning accuracy in terms of the number of landmarks, the size of the robot team, the accuracy of the robots' sensors, and the topology of the RPMG. Thus, they facilitate the prediction of the performance of a robot team in a mapping application. Certainly, the most restrictive assumption employed in the current work is that the topology of the RPMG does not change, which implies that certain pairs of robots or robots and landmarks maintain line-of-sight contact at all times. Although this is not possible in many real-world applications, the presented analysis can serve as a basis for extensions to cases where the topology of the RPMG changes dynamically. 


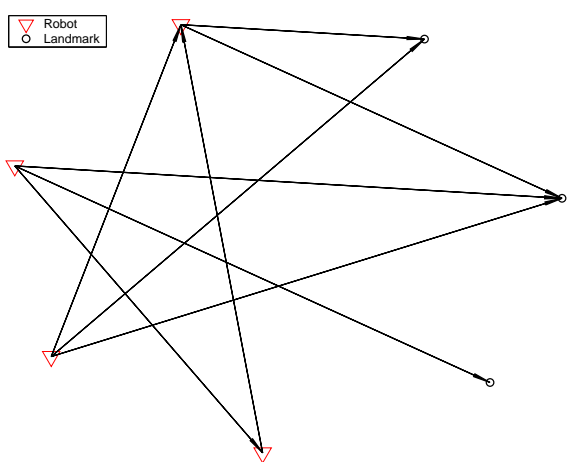

(a)

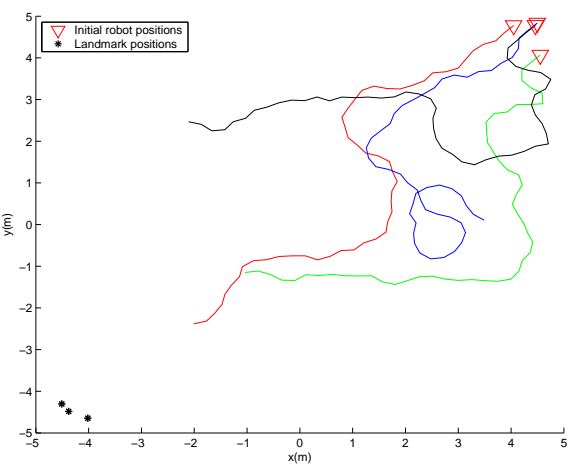

(b)

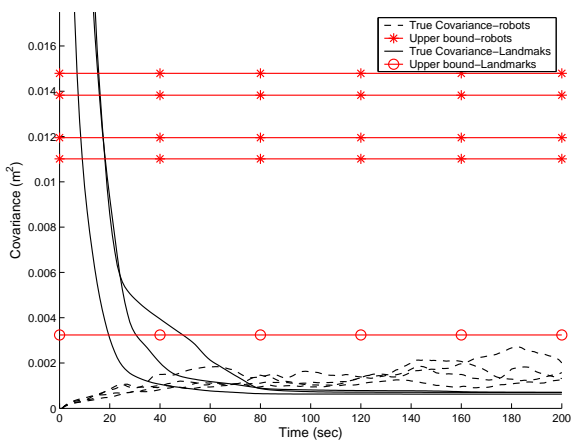

(c)

Fig. 1. (a) The RPMG used for the simulation experiments (b) The initial positions and part of the trajectories of the robots for an adverse C-SLAM scenario. (c) Comparison of the actual covariance of the position estimates against the worst-case performance bound, for the scenario in (b). The plotted lines correspond to the mean of the covariance along the two coordinate axes.

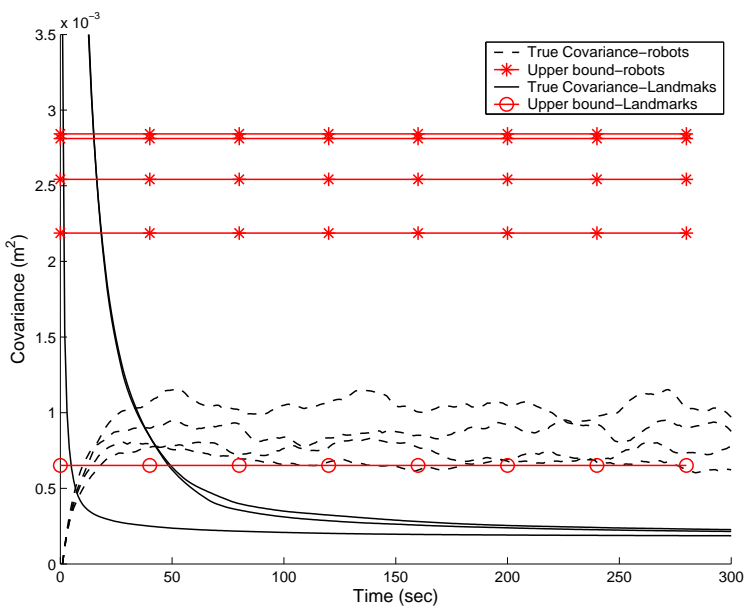

Fig. 2. Comparison of the average true covariance of the position estimates against the corresponding upper bound. Landmark positions and the initial robot positions are selected using samples from a uniform distribution Averages over 50 runs of C-SLAM are computed.

\section{ACKNOWLEDGEMENTS}

This work was supported by the University of Minnesota (DTC), the Jet Propulsion Laboratory (Grant No. 1263201), and the National Science Foundation (ITR-0324864, MRI0420836).

\section{REFERENCES}

[1] R. Kurazume, S. Nagata, and S. Hirose, "Cooperative positioning with multiple robots," in Proceedings of the IEEE International Conference in Robotics and Automation, Los Alamitos, CA, May 8-13 1994, pp. 1250-1257.

[2] S. I. Roumeliotis and G. A. Bekey, "Distributed multirobot localization," IEEE Transactions on Robotics and Automation, vol. 18, no. 5, pp. 781795 , Oct. 2002

[3] I. M. Rekleitis, G. Dudek, and E. Milios, "Multi-robot cooperative localization: A study of trade-offs between efficiency and accuracy," in Proceedings of the IEEE/RSJ International Conference on Intelligent Robots and Systems, Lausanne, Switzerland, Sep.30-Oct.4 2002, pp. 2690-2695.
[4] J. W. Fenwick, P. M. Newman, and J. J. Leonard, "Cooperative Concurrent Mapping and Localization," in Proceedings of the 2002 IEEE International Conference on Robotics and Automation, Washington D.C., 11-15 May 2002, pp. 1810-1817.

[5] R. C. Smith, M. Self, and P. Cheeseman, Autonomous Robot Vehicles. Springer-Verlag, 1990, ch. Estimating Uncertain Spatial Relationships in Robotics, pp. 167-193.

[6] P. Moutarlier and R. Chatila, "Stochastic multisensory data fusion for mobile robot location and environment modeling," in Fifth International Symposium of Robotics Research, H. Miura and S. Arimoto, Eds., Tokyo, Japan, 28-31 Aug. 1989, pp. 85-94.

[7] R. C. Smith, M. Self, and P. Cheeseman, "Estimating uncertain spatial relationships in robotics," in Proc. of the 2nd Workshop on Uncertainty in Artificial Intelligence (AAAI), Aug. 1986, pp. 1-21.

[8] J. W. Fenwick, "Collaborative Concurrent Mapping and Localization," Master's thesis, Massachusetts Institute of Technology, June 2001.

[9] M. D. Marco, A. Garulli, A. Giannitrapani, and A. Vicino, "Simultaneous localization and map building for a team of cooperating robots: a set membership approach," IEEE Transactions of Robotics and Automation, vol. 19, no. 2, pp. 238-248, Dec. 2003.

[10] S. Thrun, "A probabilistic online mapping algorithm for teams of mobile robots," International Journal of Robotics Research, vol. 20, no. 5, pp. 335-363, 2001

[11] S. B. Williams, G. Dissanayake, and H. Durrant-Whyte, "An efficient approach to the simultaneous localisation and mapping problem," in Proc. of the 2002 IEEE International Conference on Robotics and Automation, Washington, DC, May 11-15 2002, pp. 406-11.

[12] R. Madhavan, K. Fregene, and L. E. Parker, "Distributed heterogeneous outdoor multi-robot localization," in Proceedings of the 2002 IEEE International Conference on Robotics and Automation, Washington, DC May 2002, pp. 374-381.

[13] _ "Distributed cooperative outdoor multirobot localization and mapping," Autonomous Robots, Special Issue on Analysis and Experiments in Distributed Multi-Robot Systems, vol. 17, no. 1, pp. 23-39, July 2004.

[14] A. Howard, "Multi-robot mapping using manifold representations," in Proceedings of the 2004 IEEE International Conference on Robotics and Automation, New Orleans, LA, April 2004, pp. 4198-4203.

[15] A. Kelly, "General solution for linearized systematic error propagation in vehicle odometry," in Proceedings of the IEEE/RSJ International Conference on Intelligent Robots and Systems (IROS), Maui, HI, Oct.29Nov.3 2001, pp. 1938-45.

[16] A. I. Mourikis and S. I. Roumeliotis, "Performance bounds for cooperative simultaneous localization and mapping C-SLAM," Dept. of Computer Science and Engineering, University of Minnesota, Tech. Rep., 2004, http://www.cs.umn.edu/ mourikis/TR_MultiSLAM.pdf.

[17] B. Hassibi, "Indefinite metric spaces in estimation, control and adaptive filtering," Ph.D. dissertation, Stanford University, August 1996.

[18] S. Boyd and L. Vandenberghe, Convex Optimization. Cambridge University Press, 2004 